\title{
Channel Allocation Algorithm in Ad Hoc-cellular Network Based on Potential Game
}

\author{
Zengyou Sun \\ Department of Information Engineering \\ Northeast Dianli University \\ Jilin, China \\ Sunzengyou@163.com
}

\author{
Chuanhui Hao \\ Department of Information Engineering \\ Northeast Dianli University \\ Jilin, China \\ hchh518666@163.com
}

\author{
Xia Ling \\ Department of Information \\ Engineering \\ Northeast Dianli university \\ Jilin, China \\ 448131186@qq.com
}

\begin{abstract}
To reduce the interference of Ad hoc-cellular network system, and ensure the normal communication of chief users, this paper proposes an improved distributed channel allocation algorithm based on game theory. The algorithmic utility function not only considers secondary users' interference each other, but also it considers disturbance of secondary users and chief user common to other users, so it can achieve new potential function based on potential game conditions. The simulation results show that, the algorithm has better convergence, and make the chief user obtain higher SINR.
\end{abstract}

Keywords-Ad hoc-cellular network; Channel allocation; potential game;

\section{INTRODUCTION}

Game theory is the study of confrontation or competitive nature of the phenomenon of digital theory and method; it is an important subject in modern mathematics and operational research. Game theory is mainly used to study with confrontation model, to find the Nash equilibrium for the game process of the corresponding strategy, the most advantages. The literature [1] presents a channel assignment algorithm model based on game theory, the algorithm by introducing a cost function appropriate to evaluate the effects of cognitive users of the chief user; The literature [2-3] presents a channel assignment algorithm based on costs, the chief users of the algorithm for secondary users spectrum use according to certain price; The literature [5] proposed an adaptive channel allocation algorithm, the algorithm according to the selfish users and cooperative user defined 2 different objective functions; But the literature [5] proposed algorithm without considering the impact on the chief users in the network, the allocation results will make the main user obtain poor signal-to-noise ratio, which will affect the communication quality of chief users. Literature [5] proposed adaptive channel allocation algorithm on the expanding research, proposes an improved distributed channel allocation algorithm based on potential game, algorithm for interference level minimization system as the goal, the utility function of the considered time interference to chief users by households.

\section{SYSTEM MODEL}

In Ad hoc- cellular network system with $\mathrm{N}$ secondary user transmitter receiver pair, $\mathrm{M}$ main user transmitter receiver pair, the chief user and the secondary user randomly distributed in a $\mathrm{d} \times \mathrm{d}$ the region, each user has a channel, and secondary users from selecting the network channel set channel in a distributed manner. This paper assumes that the physical layer can be adjusted by the secondary user own modulation and channel coding rate to change the data transmission rate and the corresponding SINR threshold value, if the channel allocation result can not meet the primary user and the secondary user's QoS requirements, user can be satisfied the chief user and the secondary user's QoS requirements by adjusting the parameters its physical layer. Suppose in the self-organizing multi-hop Ad hoc- cellular network, the secondary user $\boldsymbol{i}$ transmitter channel gains to the user $\mathrm{j}$ receiver using $G_{i j}^{s}$, channel gain of secondary user $\boldsymbol{i}$ transmitter to the main user $m$ receiver using $G_{i m}^{s p}$,channel gain of the main user $m$ sending end to the receiving end using $G_{m m}^{p}$, the channel gain of main users' sender to the receiver using $G_{m i}^{p s}$. The formula for calculating of the channel gain is $G_{i j}=\frac{10}{d_{i j}^{2}}, d_{i j}$ is the distance of sender to receiver, namely channel gain and square of the distance is inversely , 
which is consistent with the basic characteristics of the wireless channel. In the receiving end, SINR of the secondary users $i$ is:

$$
\operatorname{SINR}_{s i}=\frac{P_{s i} G_{i i}^{s}}{\sum_{j=1, j \neq i}^{N} P_{s j} G_{i j}^{s} h\left(S_{j}, S_{i}\right)+\sum_{m=1}^{M} P_{p m} G_{m i}^{p s} h\left(S_{m}^{p}, S_{i}\right)+\sigma^{2}}
$$

In the receiving end, $S I N R$ of the main user $m$ is:

$$
\operatorname{SINR}_{p m}=\frac{P_{p m} G_{m m}^{p}}{\sum_{i=1}^{N} P_{s i} G_{i m}^{s p} h\left(S_{i}, S_{m}^{p}\right)+\sigma^{2}}
$$

In the formula: $P_{s i}$ is the transmit power of secondary users $i(i \in[1, N]) ; P_{p m}$ is the transmit power of main users $m(m \in[1, M]) ; \sigma^{2}$ is added Gauss white noise power of the receiving end; As interfere function $h$, the definition is as follows: $h\left(S_{i}, S_{j}\right)=\left\{\begin{array}{l}1, S_{i}=S_{j} \\ 0, S_{i} \neq S_{j}\end{array}\right.$

In the formula: secondary user $S_{i}$ and $S_{j}$ is channel selection strategy.

\section{POTENTIAL GAME ANALysis}

\section{A. Nash equilibrium}

The Nash equilibrium provides a prediction on the game result output, when the player $s=\left(s_{i}, s_{-i}\right)$ meet a set of strategies (3), $S$ is a Nash equilibrium.

$$
U_{i}(s) \geq U_{i}\left(s_{i}^{\prime}, s_{-i}\right), \forall i \in N, s_{i}^{\prime} \in S_{i}
$$

In the set of participants, if the participants can not change the strategy to improve their income, the whole set of participants strategies corresponding is called the Nash equilibrium. As a special game in the game theory, potential game always converges to Nash equilibrium. If potential function of game can satisfy (4), this game is a potential game.

$$
P\left(s_{i}, s_{-i}\right)-P\left(s_{i}^{\prime}, s_{-i}\right)=U_{i}\left(s_{i}, s_{-i}\right)-U\left(s_{i}^{\prime}, s_{-i}\right) s_{i}^{\prime} \in S_{i}
$$

\section{B. Utility function}

The utility function of literature [5] only considers the mutual interference between secondary users in the network, then the utility function is:

$$
U_{i}\left(s_{i}, s_{-i}\right)=-\sum_{j=1, j \neq i}^{N} P_{s i} G_{i j}^{s} h\left(s_{i}, s_{j}\right)-\sum_{j=1, j \neq i}^{N} P_{s j} G_{j i}^{s} h\left(s_{j}, s_{i}\right)
$$

This paper considers the chief users of the network in the presence of interference level, to minimize the system as the goal, so the design of the utility function must consider the interference to chief users. The utility function is proposed in this paper for :

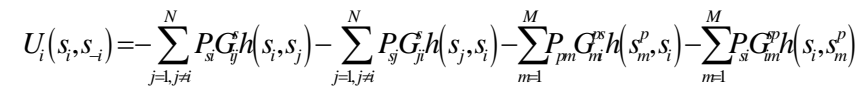

not only secondary users $I_{s s^{\prime}}=\sum_{j=1, j \neq i}^{N} P_{s i} G_{i j}^{s} h\left(s_{i}, s_{j}\right)$

and $I_{s p}=\sum_{m=1}^{M} P_{s i} G_{i m}^{s p} h\left(s_{i}, s_{m}^{p}\right)$ has the mutual interference, but the interference of chief users and other users to secondary users $I_{s^{\prime} s}=\sum_{j=1, j \neq i}^{K} P_{s j} G_{i j}^{s} h\left(s_{j}, s_{i}\right)$

and

$$
I_{p s}=\sum_{m=1}^{M} P_{p m} G_{m i}^{p s} h\left(s_{m}^{p}, s_{i}\right) .
$$

\section{Potential function}

For the utility function is proposed in this paper, construction of the potential function is:

$$
\begin{aligned}
P\left(s_{i}, s_{-i}\right) & =\frac{1}{2} \sum_{i=1}^{N}\left(-\sum_{j=1, j \neq i}^{N} p_{s i} G_{i j}^{s} h\left(s_{i}, s_{j}\right)-\sum_{j=1, j \neq i}^{N} p_{s j} G_{j i}^{s} h\left(s_{j}, s_{i}\right)\right) \\
+ & \sum_{i=1}^{N}\left(-\sum_{m=1}^{M} p_{p m} G_{m i}^{p s} h\left(s_{m}^{p}, s_{i}\right)-\sum_{m=1}^{M} p_{s i} G_{i m}^{s p} h\left(s_{i}, s_{m}^{p}\right)\right)
\end{aligned}
$$

The following paper certify that this potential function satisfies (4) type.

The utility function is expressed as: $U_{i}\left(s_{i}, s_{-i}\right)=U_{1 i}\left(s_{i}, s_{-i}\right)+U_{2 i}\left(s_{i}, s_{-i}\right)$

Inside:

$$
\begin{gathered}
U_{1 i}\left(s_{i}, s_{-i}\right)=-\sum_{j=1, j \neq i}^{N} p_{s i} G_{i j}^{s} h\left(s_{i}, s_{j}\right)-\sum_{j=1, j \neq i}^{N} p_{s j} G_{j i}^{s} h\left(s_{j}, s_{i}\right) \\
U_{2 i}\left(s_{i}, s_{-i}\right)=-\sum_{m=1}^{M} p_{p m} G_{m i}^{p s} h\left(s_{m}^{p}, s_{i}\right)-\sum_{m=1}^{M} p_{s i} G_{i m}^{s p}\left(s_{i}, s_{m}^{p}\right) \\
\text { show: } P\left(s_{i}, s_{-i}\right)=P_{1}\left(s_{i}, s_{-i}\right)+P_{2}\left(s_{i}, s_{-i}\right)
\end{gathered}
$$

Inside

$$
\begin{gathered}
P_{1}\left(s_{i}, s_{-i}\right)=\frac{1}{2} \sum_{i=1}^{N}\left(-\sum_{j=1, j \neq i}^{N} p_{s i} G_{i j}^{s} h\left(s_{i}, s_{j}\right)-\sum_{j=1, j \neq i}^{N} p_{s j} G_{j i}^{s} h\left(s_{j}, s_{i}\right)\right) \\
P_{2}\left(s_{i}, s_{-i}\right)=\sum_{i=1}^{N}\left(-\sum_{m=1}^{M} p_{p m} G_{m i}^{p s} h\left(s_{m}^{p}, s_{i}\right)-\sum_{m=1}^{M} p_{s i} G_{i m}^{s p} h\left(s_{i}, s_{m}^{p}\right)\right) \\
P_{1}\left(s_{i}, s_{-i}\right) \text {,that process has been demonstrated in }
\end{gathered}
$$
references [4],show:

$$
\begin{gathered}
P_{1}\left(s_{i}, s_{-i}\right)=-\sum_{j=1, j \neq i}^{N} p_{s i} G_{i j}^{s} h\left(s_{i}, s_{j}\right)-\sum_{j=1, j \neq i}^{N} p_{s j} G_{j i}^{s} h\left(s_{j}, s_{i}\right)+p_{1}\left(s_{-i}\right) \\
=U_{1 i}\left(s_{i}, s_{-i}\right)+p_{1}\left(s_{-i}\right)
\end{gathered}
$$

Certify: $P_{2}\left(s_{i}, s_{-i}\right)$.

$$
\begin{gathered}
P_{2}\left(s_{i}, s_{-i}\right)=\sum_{i=1}^{N}\left(-\sum_{m=1}^{M} p_{p m} G_{m i}^{p s} h\left(s_{m}^{p}, s_{i}\right)-\sum_{m=1}^{M} p_{s i} G_{i m}^{s p} h\left(s_{i}, s_{m}^{p}\right)\right) \\
=-\sum_{m=1}^{M} p_{p m} G_{m i}^{p s} h\left(s_{m}^{p}, s_{i}\right)-\sum_{m=1}^{M} p_{s i} G_{i m}^{s p} h\left(s_{i}, s_{m}^{p}\right)
\end{gathered}
$$


$+\sum_{k=1, k \neq i}^{N}\left(-\sum_{m=1}^{M} p_{p m} G_{m k}^{p s} h\left(s_{m}^{p}, s_{k}\right)-\sum_{m=1}^{M} p_{s k} G_{k m}^{s p} h\left(s_{k}, s_{m}^{p}\right)\right)$

Make:

$P_{2}\left(s_{-i}\right)=\sum_{k=1, k \neq i}^{N}\left(-\sum_{m=1}^{M} p_{p m} G_{m k}^{p s} h\left(s_{m}^{p}, s_{k}\right)-\sum_{m=1}^{M} p_{s k} G_{k m}^{s p} h\left(s_{k}, s_{m}^{p}\right)\right)$

So:

$$
P_{2}\left(S_{i}, s_{-i}\right)
$$

$=-\sum_{m=1}^{M} p_{p m} G_{m i}^{p s} h\left(s_{m}^{p}, s_{i}\right)-\sum_{m=1}^{M} p_{s i} G_{i m}^{s p} h\left(s_{i}, s_{m}^{p}\right)$

$$
+P_{2}\left(s_{-i}\right)=U_{2 i}\left(s_{i}, s_{-i}\right)+P_{2}\left(s_{-i}\right)
$$

$P_{1}\left(s_{i}, s_{-i}\right)$ and $P_{2}\left(s_{i}, s_{-i}\right)$ fed into (9) type:

$P\left(s_{i}, s_{-i}\right)=U_{1 i}\left(s_{i}, s_{-i}\right)+P_{1}\left(s_{-i}\right)+U_{2 i}\left(s_{i}, s_{-i}\right)+P_{2}\left(s_{-i}\right)$

$P_{2}\left(s_{-i}\right)$ show that the secondary users are not influenced by strategy change. When the user $\boldsymbol{i}$ becomes a potential function from the strategy $S_{i}$ to $S_{i}^{\prime}$ :

$P\left(s_{i}^{\prime}, s_{-i}\right)=U_{1 i}\left(s_{i}^{\prime}, s_{-i}\right)+P_{1}\left(s_{-i}\right)+U_{2 i}\left(s_{i}^{\prime}, s_{-i}\right)+P_{2}\left(s_{-i}\right)$

So

$$
P\left(s_{i}, s_{-i}\right)-P\left(s_{i}^{\prime}, s_{-i}\right)=\left(U_{1 i}\left(s_{i}, s_{-i}\right)+P_{1}\left(s_{-i}\right)+U_{2 i}\left(s_{i}, s_{-i}\right)+P_{2}\left(s_{-i}\right)\right)
$$

$$
-\left(U_{1 i}\left(s_{i}^{\prime}, s_{-i}\right)+P_{1}\left(s_{-i}\right)+U_{2 i}\left(s_{i}^{\prime}, s_{-i}\right)+P_{2}\left(s_{-i}\right)\right)
$$

$=\left(U_{1 i}\left(s_{i}, s_{-i}\right)+U_{1 i}\left(s_{i}, s_{-i}\right)\right)-\left(U_{1 i}\left(s_{i}, s_{-i}\right)+U_{1 i}\left(s_{i}, s_{-i}\right)\right)$

$$
=U_{i}\left(s_{i}, s_{-i}\right)-U_{i}\left(s_{i}^{\prime}, s_{-i}\right)
$$

So this game is a potential game, and the game will converge to a Nash equilibrium.

\section{AlgORITHM STEPS}

\section{A. Initialize}

Channel and transmitting power are distributed into users by random.

\section{B. Iteration}

1) Secondary users shall be game in accordance by the order of access network.

2) Sender of secondary user $i$ calculate $I_{\text {ss }}$ and $I_{s p}$, and the computed value tells the receiver by the common control channel.The receiver calculate $I_{s ' s}$ and $I_{p s}$,so the received $I_{s s}$ and $I_{s p}$ could compute each of channel utility function $U_{i}\left(s_{i}, s_{-i}\right)$.

3) The receiving end of secondary user $i$ selects maximum channel by utility function, and tell its transmission by the common control channel, then secondary users'new strategy is for $s_{i}^{\prime}=\arg \max \left(U_{i}\left(s_{i}, s_{-i}\right)\right)$.

4) The iterative process is repeat until convergence.

\section{SIMULATION ANALYSIS}

It randomly deployed $N=40$ secondary user transmitter-receiver pairs and $M=7$ primary user transmitter-receiver pairs in the $200 \times 200$ region. Maximum distance of transmission and receiver is $50 \mathrm{~m}$ between chief users and secondary users. The transmit power of secondary users and chief users are $[1,3] m w$ and $[2,5] m w$, then noise power is $\sigma^{2}=10^{-9} \mathrm{mw}$.

\section{A. Algorithm Convergence}

Through channel allocation algorithm based on potential game, each user policy change is figure 2 .

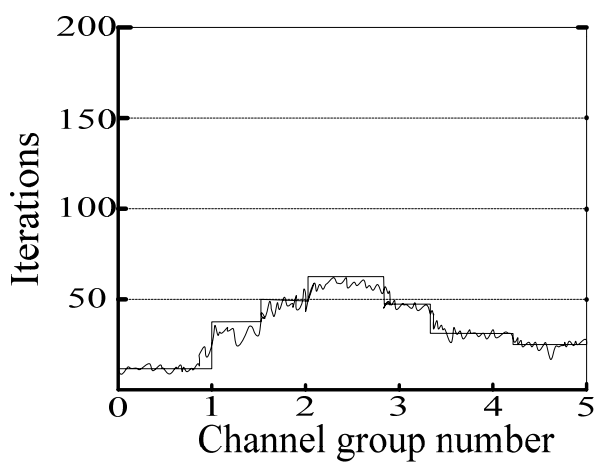

Fig. 1 user policy convergence

In figure 1, when the iterative algorithm is about 60 times, the secondary user strategy does not change, which reaches the equilibrium state. It shows that the algorithm has better convergence.

\section{B. Algorithm Reliability Comparison}

By the reference [6], adaptive channel allocation SIRN:

$\operatorname{SINR}^{m}(k)=\left|h^{m}(k)\right|^{2} \cdot \rho$.Assuming that each subcarrier average signal power of each antenna is for $\mathrm{P}$, defined $\rho=P / \sigma^{2}$ as the average transmit SIRN. $h^{m}(k)$ is transmitting antenna to the user M'receiving antenna channel subcarrier $\mathrm{K}$ response. In different period of time, the bandwidth is $30 \mathrm{GHz}$, and carrier number is 1024, and the noise power is $\sigma^{2}=10^{-9} \mathrm{mw}$. By simulation comparison, SIRN of potential game channel allocation algorithm is higher than the proposed numerical adaptive channel allocation algorithm of SINR values. 


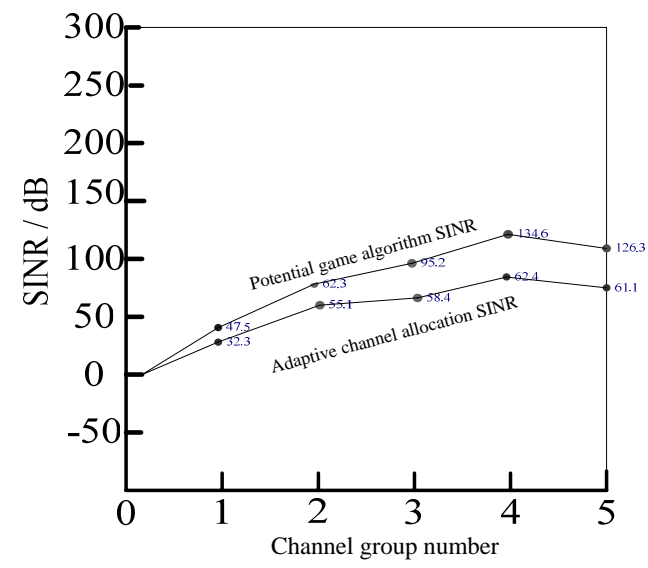

Figure 2 SINR size variation

\section{CONCLUSION}

This paper presents a new channel assignment algorithm for improving Ad hoc-cellular network system. In the Ad hoc-cellular networks, chief users and secondary users exist at the same time, based on this, this paper considers the interference to the chief user of the times in the utility function. At the same time, interference situation is maximized both the secondary user and chief user by their own utility. It is proved the game model of the potential game, and simulation results show the convergence property of the proposed algorithm and higher SIRN.

\section{REFERENCES}

[1] Xiaolin Li, Haitao Liu. Cognitive radio spectrum allocation algorithm model based on Game Theory[J].Journal of Chongqing University of Posts and Telecommunications: Natural Science Edition,2010,22(2):151-155.

[2] NIYATO D. HOSSAIN E.A Game-Theoretic Approach to Competitive Spectrum Sharing in Cognitive Radio Networks[C]//IEEE WCNC. Hong Kong: IEEE press, 2007: 16-20.

[3] NIYATO D, HOSSAIN E. Competitive Spectrum Sharing in Cognitive Radio Networks: A Dynamic Game Approach[J].IEEE Transaction on Wireless Communications， 2008， 7(7):2651-2660.

[4] WU Yong-le. WANG Bei. Repeated Open Spectrum Sharing Game with Cheat-Proof Strategies [J].IEEE Transaction on Wireless Communications, 2008, 8(4):1922-1933.

[5] NIE N.COMANICIU C. Adaptive channel allocation spectrum etiquette for cognitive radio networks[C]//IEEE DySPAN, 2005. Baltimore: IEEE press, 2005:269-279.

[6] Guanding Yu, Zhaoyang, Zhang Lai Ping .The multi antenna OFDM systems with adaptive sub channel allocation and antenna selection [J]. Journal of circuits and systems , 2005,10(6):63-68

[7] MONDERER D,SHAPLEY L S, Potential game [J]Journal of Games and Economic Behavior, 1996, 14(3):124-143. 